\title{
Enhanced Raman microprobe imaging of single-wall carbon nanotubes
}

\author{
V G Hadjiev ${ }^{1}, \mathrm{~S} \mathrm{Arepalli}^{2}, \mathrm{P}$ Nikolaev $^{2}, \mathrm{~S} \mathrm{Jandl}^{3}$ and L Yowell ${ }^{4}$ \\ ${ }^{1}$ Texas Center for Superconductivity and Advanced Materials, University of Houston, \\ Houston, TX 77204, USA \\ ${ }^{2}$ GB Tech./NASA-Johnson Space Center, 2101 NASA Road One, Houston, TX 77058, USA \\ ${ }^{3}$ Department of Physics, University of Shertrooke, Sherbrooke, PQ, J1K 2R1, Canada \\ ${ }^{4}$ NASA-Johnson Space Center, 2101 NASA Road One, Houston, TX 77058, USA
}

Received 19 August 2003

Published

Online at stacks.iop.org/Nano/15/1 (DOI: 10.1088/0957-4484/15/0000)

\begin{abstract}
We explore Raman microprobe capabilities of visualizing single-wall carbon nanotubes (SWCNTs). Although this technique is limited to the micron scale, we demonstrate that images of individual SWCNTs, bundles, or their agglomerates can be generated by mapping Raman active elementary excitations. We measured the Raman response from carbon vibrations in SWCNTs excited by confocal scanning of a focused laser beam. Carbon vibrations reveal key characteristics of SWCNTs such as the nanotube diameter distribution (radial breathing modes (RBM), $100-300 \mathrm{~cm}^{-1}$ ), the presence of defects and functional groups (D-mode, $1300-1350 \mathrm{~cm}^{-1}$ ), strain and oxidation states of SWCNTs, as well as the metallic or semiconducting character of the tubes encoded in the lineshape of the G-modes at $1520-1600 \mathrm{~cm}^{-1}$. In addition, SWCNTs are highly anisotropic scatterers. The Raman response from a SWCNT is maximal for incident light polarization parallel to the tube axis and vanishing for perpendicular directions. We show that the SWCNT bundle shape or direction can be determined, with some limitations, from a set of Raman images taken for two orthogonal directions of the incident light polarization.
\end{abstract}

(Some figures in this article are in colour only in the electronic version) 


\title{
Enhanced Raman Microprobe Imaging of Single Wall Carbon Nanotubes
}

\author{
V. G. Hadjiev ${ }^{1}$, S. Arepalli², P. Nikolaev² ${ }^{2}$ S. Jandl ${ }^{3}$, and L. Yowell ${ }^{4}$ \\ ${ }^{1}$ Texas Center for Superconductivity and Advanced Materials, \\ University of Houston, Houston, Texas 77204 \\ 2 G. B. Tech./NASA-Johnson Space Center, \\ 2101 NASA Road One, Houston, TX 77058 \\ ${ }^{3}$ Department of Physics, University of Sherbrooke, Sherbrooke, PQ J1K 2R1, Canada \\ 4 NASA-Johnson Space Center, 2101 NASA Road One, Houston, TX 77058
}

(Dated: December 15, 2003)

\begin{abstract}
We explore Raman microprobe capabilities to visualize single wall carbon nanotubes (SWCNTs). Although this technique is limited to a micron scale, we demonstrate that images of individual SWCNTs, bundles or their agglomerates can be generated by mapping Raman active elementary excitations. We measured the Raman response from carbon vibrations in SWCNTs excited by confocal scanning of a focused laser beam. Carbon vibrations reveal key characteristics of SWCNTs as nanotube diameter distribution (radial breathing modes, RBM, 100-300 $\mathrm{cm}^{-1}$ ), presence of defects and functional groups (D-mode, $1300-1350 \mathrm{~cm}^{-1}$ ), strain and oxidation states of SWCNTs, as well as metallic or semiconducting character of the tubes encoded in the lineshape of the Gmodes at $1520-1600 \mathrm{~cm}^{-1}$. In addition, SWCNTs are highly anisotropic scatterers. The Raman response from a SWCNT is maximal for incident light polarization parallel to the tube axis and vanishing for perpendicular directions. We show that the SWCNT bundle shape or direction can be determined, with some limitations, from a set of Raman images taken at two orthogonal directions of the incident light polarization.

PACS numbers: 78.30.Na, 78.67.Ch, 42.30.Va
\end{abstract}




\section{INTRODUCTION}

Single wall carbon nanotubes (SWCNTs) have been receiving increased interest for applications exploiting their individual properties. Some of these properties are unique as the high aspect ratio (small diameter of $\sim 1 \mathrm{~nm}$ and long length of many microns), high thermal and chemical stability, very good heat conduction, and high mechanical strength (elastic modulus of $\sim 1 \mathrm{TPa}$, comparable to that of diamond).[1] The electronic properties of nanotubes depend on the manner in which the graphene sheet is wrapped up to form a nanotube (tube diameter and chirality). SWCNTs can be metallic, semiconducting with a very small energy gap (a few meV) or semiconducting with a moderate energy gap (few tenths of eV). Conductivity measurements on individual SWCNTs have shown rectification effects for some nanotubes and ohmic conductance for others. These properties suggest that nanotubes could lead to a new generation of nanoscopic electronic devices.[2] A reproducible implementation of SWCNTs in nanoscopic devices requires effective control by means of imaging techniques. Although some of the available techniques as Atomic Force Microscopy (AFM) are very helpful and cost effective, if compared to electron microscopy (EM) and scanning tunneling microscopy (STM), they probe only part of the important properties of the carbon nanotubes. In contrast, spectroscopic characteristics of the carbon nanotubes reflect most of their physical properties. The nanotube diameter and chirality uniquely determine the carbon vibrations (having phononic character) and nanotube electronic structure. Among the spectroscopic techniques only Raman scattering utilizes to full extent the peculiar interplay of SWCNTs properties.[3] Raman scattering from phonons in SWCNTs is mediated by electrons via electron-phonon coupling thus probing the electronic structure as well. One dimensional character of SWCNTs give rise to sharp van Hove singularities (vHS) in the electronic density of states (eDOS), which leads to a strong resonant Raman scattering process.[4] As a result, Raman scattering response of SWCNTs depends on the tube diameter, $[5,6]$ tube axis direction, $[7]$ chirality, and electronic character of excited nanotubes.[8, 9] The resonant character of Raman scattering increases the scattering efficiency to a level that an individual SWCNTs can be detected.[10] The specificity of the Raman spectroscopy of SWCNTs suggests that a Raman imaging technique would be a highly effective imaging tool.

In this article we explore the capabilities and limitations of confocal Raman imaging 


\section{ides a methodology}

technique to locate and characterize isolated or agglc

technique to locate and characterize isolated or agglomerated SWCNTs having submicron size. The main ideology is to couple light that inherently allows micron resolution to excitations that are confined to individual carbon nanotubes with nanometer size. This coupling is realized in Raman scattering process. We show that the Raman imaging of vibrations in SWCNTs could compensate, to some extent, the diffraction limited resolution of optical microscopy by measuring additional to spatial properties of SWCNTs. The Raman imaging technique described herein is akin to the Raman chemical imaging but instead of mapping different chemical components in the sample we produce images of different vibrations of SWCNTs, which in turn reflect specific nanotube properties. Recently, diffraction limited Raman imaging and corresponding AFM images of isolated SWCNTs and thin bundles have been reported.[11] The approach in Ref. 11 generates high quality images based on integrated Raman intensity over frequency range containing several carbon vibrations. In this case the Raman image is more topographic than physically and chemically distinguishable. Further increase of the Raman imaging resolution to nanoscale has been achieved by mapping surface enhanced Raman scattering (SERS) induced by scanning a sharp metallic tip over the carbon nanotubes.[12] SERS, however, is a complex excitation of SWCNTs that involves surface plasmons of the scanning tip and one needs more investigation on how to relate SERS to the spontaneous Raman spectra. Both techniques[11, 12] require specific modifications of standard Raman spectroscopy systems. Instead, we demonstrate Raman imaging in details using a commercial spectrometer that otherwise is used to cover a broad range of analytical applications.

\section{RAMAN IMAGING TECHNIQUE: EXPERIMENTAL DETAILS}

In the Raman imaging experiment we scanned SWCNTs deposited on a quartz plate by means of a pulsed-laser vaporization technique. Briefly, the deposition setup uses a doublepulse laser oven process for nanotube production. Small quartz flats (6mm in diameter) are placed inside the flow tube at different distances from the target. In order to limit the nanotube production to individual tubes and small diameter bundles, the laser ablation was run for only $0.5 \mathrm{sec}$ while maintaining other normal production conditions (such as temperature of $1473 \mathrm{~K}$ in argon flow and laser energy density of $1.5 \mathrm{~J} / \mathrm{cm}^{2}$ ). The length and diameter distribution of the isolated SWCNTs and small bundles is carried out using 
an AFM in tapping mode. Some of the areas on these quartz flats that show clear images of isolated tubes are marked and are used for the current Raman study. More details of the sample preparation are given in Ref.13

The Raman measurements were performed on a LabRam HR (Jobin Yvon, Horiba), 800 mm focal length, multichannel spectrometer equipped with a microscope. The spectrometer was set up in a line scan imaging configuration that takes full advantage of the two dimensional CCD detector having 2000 pixels along the spectral dispersion and 800 pixels along the slit height. A microscope $100 \times$ objective focuses the light beam of a HeNe $(632.8 \mathrm{~nm})$ laser on the sample surface. The laser spot size $d_{l} \simeq 1.15 \mu \mathrm{m}$ is estimated from the Gaussian optics expression $d_{l}=4 \lambda f / \pi D_{1 / e^{2}}$, where $\lambda=0.6328 \mu m, f=1.8 \mathrm{~mm}$ (objective 100X) and $D_{1 / e^{2}}=1.26 \mathrm{~mm}$ is the laser beam diameter. The $d_{l} \simeq 1.15 \mu \mathrm{m}$ laser spot creates a circular image with a diameter of 20 pixels on the CCD. The relation 20 pixels $=d_{l} \simeq 1.15 \mu \mathrm{m}$ gives the scaling factor that allows us to relate distances on the image to those on the sample surface. In the line scan imaging regime of LabRam HR, an optical scanner deflects the focused laser beam in a manner that creates a row of laser spots arranged in a line on the sample. In Fig. 1 (a) we show an optical microscope image of the sample with "line" focused laser beam on the surface. This line is then projected along the entrance slit height of the spectrometer (stigmatic type). On the CCD, the line image is projected along the 800 pixels direction and recorded at 5 pixels binning, whereas the spectra were dispersed along the 2000 pixels (12 pixels binning) edge of the CCD. The particular line scan shown in Fig. 1 (a) excited the spectral image displayed in Fig. 1 (b). The spectrometer was tuned to cover the spectral range of 1350 to $1850 \mathrm{~cm}^{-1}$. Figure 1 (b) shows that the line scan covering $\approx 20 \mu \mathrm{m}$ (400 pixels) clearly crosses at least five SWCNTs or bundles. Such a 3D image is very informative. It gives the relative position of the SWCNTs on the line scan trace and the spectral data for each of the Raman scatterers. The raster scan of the sample surface was achieved by moving the microscope table in a direction perpendicular to the line scan at $\approx 1 \mu \mathrm{m}$ steps thus recording a series of consecutive images as that one shown in Fig. 1 (b). The Raman data collected from the scan contains both spatial and spectroscopic information. Firstly, we analyze the line scan Raman data. The spatial part of the data corresponds to an optical image as the one shown in Fig. 1 (a) divided into cells of size $\left(d_{l} / 4\right) \times d_{l}$ or $5 \times 20$ pixels image on the CCD. Each Raman spectrum in Fig. 1 (b) is generated by a single cell, that is, each cell acts as a Raman scatterer. In our experiment every line scan was recorded 
in parallel incident and scattered light polarizations for two orthogonal directions for the incident light polarization.

\section{MAPPING CODES FOR SINGLE WALL CARBON NANOTUBES}

The Raman microprobe scanning technique described in the previous section generates a cellular grid type of data base, a Raman microprobe map. Each cell on the map determines an original pixel on the Raman microprobe image. In order to create an image of the scanned area we have to code these cells with colors or levels of grey that correspond to certain spectroscopic characteristics. Examples of mapping codes are the integrated Raman intensity under certain Raman peaks or the frequency of a given vibration. The latter may vary in different parts of the sample due to non-homogeneous stress distribution or chemical modification. In this section we list the salient Raman scattering properties of SWCNTs that suggest rich variety of mapping codes.

The key Raman feature of SWCNTs is the extremely strong Raman scattering rate when the incident or scattered photon is in resonance with interband transitions between vHS.[10] The resonant Raman effect makes feasible Raman imaging of a single carbon nanotube and reveals unique information about both the electronic and vibrational properties of SWCNTs. Tight-binding calculations provide the simplest approach to the electronic band structure of SWCNTs.[14] Quasi 1D character of SWCNT tubular structure results in a very large electronic density of states at vHS energy that depends on the tubule diameter $d_{t}$. Interband transitions between vHS in semiconducting SWCNT vary with $d_{t}$ as: $E_{11}^{S}=2 \gamma_{0} a_{C-C} / d_{t}$ between the first pair of vHs, and $E_{22}^{S}=2 E_{11}^{S}, E_{33}^{S}=4 E_{11}^{S}$ for the second and third ones, respectively, where $\gamma_{0}=2.9 \mathrm{eV}, a_{C-C}=0.142 \mathrm{~nm}$. The first interband transition for the metallic nanotubes is at $E_{11}^{M}=6 \gamma_{0} a_{C-C} / d_{t}$. More precise ab initio calculations give deviations from these simple expressions only for small diameter $(<1 \mathrm{~nm})$ nanotubes.[15] Here comes the first important characteristic of Raman scattering from SWCNTs, namely that laser excitation with a given wavelength excites only a subset of all nanotubes that The secen inpont chasoitent if.

may present in the sample. Next one is that optical transitions between vHS are strongly polarized. They can be generated only if the incident light has electric field polarization component along the nanotubes axis. It is well established experimentally that Raman intensity of SWCNTs measured for parallel incident $(I)$ and scattered $(S)$ light polarizations 
vary with the angle $\theta$ between the light polarizations $\vec{e}_{I, S}$ and the nanotube axis as $\cos ^{4} \theta .[7]$

We briefly survey the origin of carbon vibrations in SWCNTs. There are four basic groups of Raman active vibrations in SWCNTs that reveal distinctive structural and electronic properties. The in-phase vibration of carbon atoms in direction perpendicular to the nanotube axis, called the radial breathing mode (RBM), is usually observed between 100 and $300 \mathrm{~cm}^{-1}$ in the Raman spectra. Similarly to the interband transition energy, the RMB frequency $\omega_{R M B}$ is proportional to $1 / d_{t}$. [16] The Raman measurements of this mode allow determination of the diameter distribution of the subset of SWCNTs that is in resonance with the laser excitation. Mapping of the RMB modes Raman intensity can visualize the diameter distribution of SWCNTs over the scanned area. In the present work, we do not explore this possibility because of the relatively weak RBM intensity and the time restriction imposed over our experiment. The RBM mapping, however, is feasible and could create very informative images in particular when multiple laser excitation is used.

Next important group of carbon vibrations is that of the "disorder induced", D-band. The first-order Raman scattering from materials with translational symmetry as SWCNTs along the nanotube axis is restricted to the long wavelength vibrations (with wavevector $\mathbf{q} \approx 0$ ) because of the requirement for the quasi-momentum conservation in the scattering process. In graphite, the in-plane "breathing" vibrations of carbon hexagons have wavevectors around the K-point in the Brillouin zone and are Raman forbidden. However, disorder in graphite facilitates a peculiar double resonant process that makes these vibrations Raman active.[17] The Raman feature corresponding to these vibrations is usually called the D-band. This band is also observed in "defect" SWCNTs and similarly to the D-band in graphite it is highly dispersive: the D-band frequency, $\omega_{D}$ increases linearly with the laser excitation energy in the range $1290-1360 \mathrm{~cm}^{-1} \cdot[3,18]$ The Raman scattering from the second harmonic of the D-band, the $\mathrm{D}^{\star}$-band, is always allowed and needs no disorder to be observed.[19]

Finally, we describe the properties of the tangential C-C bond stretching vibrations of SWCNTs having frequencies in the range $1520-1600 \mathrm{~cm}^{-1}$.[3] These modes comprise the Gband of SWCNTs, called after the original $E_{2 g_{2}}$ vibration at $1582 \mathrm{~cm}^{-1}$ in graphite. Usually the G-band profile for SWCNTs nanotube bundles can be deconvoluted into four spectral lines at $\sim 1540, \sim 1570, \sim 1590$, and $\sim 1610 \mathrm{~cm}^{-1}$. The analysis of these lines is complicated by the superimposed Raman features of nanotubes with different diameters and chirality that are presented in the bundles. Nevertheless, some important observations have been done 
under resonance with specific group of nanotubes. For instance, the G-band at resonance with semiconducting SWCNTs exhibits a sharp peak at $\sim 1590 \mathrm{~cm}^{-1}$ and a weaker sideband within $1560-1570 \mathrm{~cm}^{-1}$. The profile of the G-band of metallic nanotubes is dominated by a broad feature $\sim 1540 \mathrm{~cm}^{-1}$, having sometimes apparent asymmetric lineshape, and a much sharper peak at $\sim 1580 \mathrm{~cm}^{-1}$. [8] The $632.8 \mathrm{~nm}$ laser line excites mettalic tubes with $d_{t} \approx 1.30$.[4] Recent extensive Raman study[6] of various isolated SWCNTs has revealed that the G-band of a SWCNT is essentially a doublet with a higher frequency component $\omega_{G}^{+}$independent of $d_{t}$ and the low frequency component $\omega_{G}^{-}=\omega_{G}^{+}-C / d^{\alpha}$, with $\alpha \approx 1.4$ [20] and $C$, a constant that depends on the symmetry of the mode and whether the tube is semiconducting or metallic.

In the present work we derive the mapping codes from the spectroscopic characteristics of the G-band. This band is the most informative vibrational group of SWCNTs. The frequency component $\omega_{G}^{-}$is tube diameter dependent and it is strongly renormalized in the metallic SWCNTs featuring a huge broadening. The higher frequency component $\omega_{G}^{+}$ gives important information about the charge and stresses on the nanotube. Oxidation of SWCNTs leads to upward shift of $\omega_{G}^{+}$by $320 \mathrm{~cm}^{-1}$ per hole, per carbon atom.[21] Reduction, as for instance $\mathrm{K}$ or Rb doping of SWCNTs, has opposite effect on $\omega_{G}^{+}$. [22] Compressive axial strain of SWCNTs results in increasing of $\omega_{G}^{+}$by $15 \mathrm{~cm}^{-1} / \%$.[23]

\section{RAMAN IMAGE FORMATION}

In Sections II and III we described the key ingredients of the Raman image formation. These are the cellular grid that covers the scanned portion of the sample surface with associated Raman spectra and the mapping codes. Firstly, we proceed with the line scan shown in Fig. 1 (b). It contains 112 Raman spectra covering the spectral range of the Dband and G-band vibrations of SWCNTs. Two of these spectra are shown in Fig. 2. Each spectrum presents the Raman response from a scatterer with size $0.3 \times 1.2 \mu m^{2}$ (see Section II) on the sample surface. Next we define the mapping code to be the integrated intensity under the Raman peaks. In the simplest approach this can be accomplished by summation of the spectral weight below the curve over certain spectral range. For instance, in the present work we use three mapping codes that correspond to the integrated Raman intensity of the D-band, $\omega_{G}^{-}$, and $\omega_{G}^{+}$bands over the spectral ranges marked by the vertical dash-lines in 
Fig. 2. Having defined the mapping code as integrated Raman intensity, in the next step we plot the variation of the Raman intensity along the line scan. In Fig. 3 we present $\omega_{G}^{+}$-band profile of the line scan shown in Fig. 1 (b). With this step we assign the quantity Raman intensity to definite spot on the scanned surface. The peaks in Fig. 3 correspond to spots on the scan where the laser beam hit SWCNTs or bundles. Note that the linewidth of the peaks is at best 20 pixels, that is, the spatial resolution is convoluted by the size of the focused laser spot. Finally, the two dimensional (2D) image of the scanned area is assembled from Raman intensity profiles as the one in Fig. 3 acquired for consequent positions of the line scan shifted at $1 \mu \mathrm{m}$ steps in the direction perpendicular to the line image. Thus created 2Dimage is characterized by different degree of seamlessness along and perpendicular to the line scan. The cell size along the line scan is submicron, $d_{l} / 4$, whereas in perpendicular direction is $d_{l} \approx 1.15 \mu \mathrm{m}$. As we showed above the lower than $d_{l}$ pixel size gives no enhancement of the resolution but improves the image seamlessness. To achieve the similar seamlessness in the perpendicular direction we applied a bilinear interpolation on the original $d_{l} / 4 \times d_{l}$ cell based image and transformed it to $\bar{d}_{l} / 4 \times d_{l} / 4$ type one. This operation fits a bilinear surface through existing data points (cells). The value of the interpolated points is a combination of the values of the four closest points.[24] In Fig. 4 we present the Raman images $\left(d_{l} / 4 \times d_{l} / 4\right.$ pixel size) of $12 \times 9 \mu \mathrm{m}^{2}$ area, covered by SWCNTs for the three mapping codes. We can identify areas with predominately one type of tubes, semiconducting or metallic, simply by comparing $\omega_{G}^{+}$and $\omega_{G}^{-}$-band images displayed in Fig. 4. Going back to Fig. 2 we note that the Raman spectrum having strong and broad $\omega_{G}^{-}$-band steams from a bundle containing metallic tubes, whereas the other, overall much weaker, spectrum is possibly from an isolated semiconducting SWCNT. Qualitatively, areas having stronger $\omega_{G}^{+}$than $\omega_{G}^{-}$-band intensity are most likely deposited by semiconducting SWCNTs. To support this criteria, we present in Fig. 5 the Raman spectra corresponding to the spots 1-5 on the Raman image in Fig. 4 (b). The Raman lineshape in Fig. 5-1 clearly indicates a presence of a semiconducting SWCNT. The strong overall signal from the spot 4 reveals a relatively thick bundle of mostly metallic SWCNTs at that place, an assignment further supported by the obvious softening of the $\omega_{G}^{+}$band in Fig. 5-4.[9] The area around the spot 5 contains âgain mixed type SWCNT bundles but with predominately semiconducting nanotubes as revealed in Fig. 5-5. Now we analyze the D-band image in Fig. 4 (c). The non-vanishing intensity of the D-band is indication fer defects on the SWCNTs. Interestingly, the nanotubes on the area covering the 
spots 1-3 exhibit no appreciable D-band intensity, whereas the remaining part of the D-band image in Fig. 4 (c) is reproducing the images in Figs. 4 (a) and (b). On the other hand, the relatively low Raman intensity at spots 1-3 in Fig. 4 (a) and (b) suggests that corresponding area is covered mostly by isolated SWCNTs. This leads us to the conclusion that during the growth process individual SWCNTs got less damaged than nanotubes grown in bundles or/and nearest neighboring SWCNTs in the bundle may facilitate the double resonance process as well.

\section{LINEARLY POLARIZED IMAGING: IMPROVING RESOLUTION}

In this section we explore the opportunity to improve the resolution of the SWCNTs images by reconstructing the nanotube dimensions, especially the length. The Raman intensity of a SWCNT, measured for parallel incident and scattered light polarizations, varies as $I_{S}=I_{0} \cos ^{4} \theta$ with the angle $\varphi$ between the polarization vectors $\vec{e}_{I, S}$ and the nanotube axis.[7] We can use this relation to construct the image of angle distribution $\theta(x, y)$ over the scanned areag that is, each pixel will be given the value of the angle between SWCNTs and the polarization vectors. In our experiment we recorded polarized Raman spectra of the scanned area for two directions of the light polarization: along and perpendicular to the line scan. For instance, the images shown in Fig. 4 are derived from the Raman spectra taken with $\vec{e}_{I, S}$ along the horizontal edge of the image. Let us denote the Raman intensity at $(x, y)$ point on the image as $I_{h}(x, y, \theta)=I_{0} \cos ^{4}(\theta)$ for $\vec{e}_{I, S}$ along the horizontal direction. The corresponding expression for $\vec{e}_{I, S}$ pointing the vertical direction is then $I_{v}(x, y, \theta)=I_{0} \cos ^{4}(\pi / 2-\theta)$. The angle $\theta$ can be easily found from these expressions and is given by $\theta(x, y)=\operatorname{arctg} \sqrt[4]{I_{v}(x, y) / I_{h}(x, y)}$. Note that one obtains the same result also for a tube at angle $-\theta$, that is, there is inherent uncertainty to determine the tube direction only from the Raman spectra provided the length of the nanotube is shorter than $d_{l}$. Another limitation comes from overlapped SWCNTs having different orientations. To illustrate the angle distribution imaging we further analyze the framed area on the Raman image in Fig. 4 (a). The image in Fig. 4 (b) that contains the spot 1-3 along with the corresponding spectra in Fig. 5 indicate a semiconducting SWCNT stretched between spot 1 and 2 with length $>d_{l}$ and overlapping metallic and semiconducting nanotubes at spot 3. In Fig. 6 (a) and (b) we display the $\omega_{G}^{+}$images covering the framed area in Fig. 4(a) for the vertical 
and horizontal directions of $\vec{e}_{I, S}$, respectively. The very intense image taken for horizontal direction of $\vec{e}_{I, S}$ suggests that the direction of the SWCNTs is closer to horizontal than to vertical one $\left(\theta<45^{\circ}\right)$. The horizontally polarized $\omega_{G}^{-}$image shown in Fig. $4(\mathrm{~b})$ and the one for vertical polarization (not shown here) exhibit similar the to $\omega_{G}^{+}$images contrast. One way to make an image of the angle distribution is to plot the iso-angle contours. In Fig. 6 (c) we present the SWCNTs angle distribution derived from the images given in Fig. 6 (a) and (b). To improve the visualization of the angle distribution in Fig. 6 (c) we decorated the image by sketching the possible position of the nanotubes. Note that the orientation of the SWCNT on the upper part of the image cannot be determined because of the short nanotube length.

\section{CONCLUSIONS}

In this work we demonstrate the capabilities of the Raman imaging to locate and identify isolated SWCNTs or bundles deposited on non-metallic surface. Although the spatial resolution is limited by the size of the point illumination, we reveal more details on some of the images using a decorative technique based on the collected spectral data. Extensive fit treatment of the Raman spectra implemented in the Raman image, though being a tedious work, would add such a subtle but important detail as stress/charge state of the SWCNT object.

\section{Acknowledgments}

This work is supported in part by the State of Texas through the Texas Center for Superconductivity and Advanced Materials, the Texas Institute for Intelligent Bio-Nano Materials and Structures for Aerospace Vehicles, funded by NASA Cooperative Agreement No. NCC-1-02038, and Lockheed Martin contract NAS9-19100.

[1] For review see "Carbon Nanotubes, Synthesis, Structure, Properties, and Applications," Dresselhaus MS, Dresselhaus G, Avouris P Eds. 2000 in Topics in Applied Physics 80

[2] Collins PG, Zettl A, Bando A, Thess A, Smalley RE 1997 Science bf 278100

[3] Dresselhaus MS and Eklund PC 2000 Adv. Phys. 49705 
[4] Kataura H, Kumazawa Y, Maniwa Y, Umezu I, Suzuki S, Ohtsuka Y and Achiba Y 1999 Synth. Metals 1032555

[5] Rao AM, Richter E, Bandow S, Chase B, Eklund PC, Williams KA, Fang S, Subbaswamy KR, Menon M, Thess A, Smalley RE, Dresselhaus G and Dresselhaus MS 1997 Science 275 187

[6] Jorio A, Souza Filho AG, Dresselhaus G, Dresselhaus MS, Swan AK, Ünlü MS, Goldberg BB, Pimenta MA, Hafner JH, Lieber CM and Saito R 2002 Phys. Rev. B 65155412

[7] Gommans HH, Alldredge JW, Tashiro H, Park J Magnuson J amd Rinzler AG 2000 J. of Appl. Phys. 882509

[8] Brown SDM, Jorio A, Corio P, Dresselhaus MS, Dresselhaus G, Saito R and Kneipp K 2001 Phys. Rev. B 63155414

[9] Dubay O, Kresse G and Kuzmany H 2002 Phys. Rev. Lett. 88235505

[10] Dresselhaus MS, Dresselhaus G, Jorio A, Souza AG and Saito R 2002 Carbon 402043

[11] Mews A, Koberling F, Basche T, Philipp G, Duesberg GS, Roth S, Burghard M 2000 Adv. Materials 12, 1210

[12] Hartschuh A, Sanchez AJ, Xie XS and Novotny L 2003 Phys. Rev. Lett. 90095503

[13] Apepalli S, Nikolaev P, Holmes W and Files BS 2001 Appl. Phys. Letters 781610

[14] Saito R, Dresselhaus G and Dresselhaus MS 1998 Physical Properties of Carbon Nanaotubes (Imperial College Press, London)

[15] Gülseren O, Yildirim T and Ciraci S 2002 Phys. Rev. B 65153405

[16] The RMB mode has $A_{1(g)}$ symmetry. It is an even (g, gerade) mode only in achiral, armchair and zig-zag, SWCNTs. Generally, the RBM frequency depends on with the nanotube diameter $d_{t}$ as $\omega_{R M B}\left(\mathrm{~cm}^{-1}\right)=A\left(\mathrm{~cm}^{-1}\right) / d_{t}(\mathrm{~nm})+B\left(\mathrm{~cm}^{-1}\right)$. The RMB frequency of an insulated SWCNT is well expressed with $B=0$, whereas in a bundle it is modified due to the inter tube interactions. The latter requirs a modification of $A$ in the expression for $\omega_{R M B}\left(\mathrm{~cm}^{-1}\right)$ and $B \neq 0$.

[17] Thomsen C and Reich S 2000 Phys. Rev. Lett. 855214

[18] Maultzsch J, Reich S and Thomsen C 2001 Phys. Rev. B. 64121407

[19] In this second-order Raman process the momentum conservation is always obeyed because the scattering invoves two D-band phonons with opposite wavevectors, that is, with resulting wavevector $\approx 0$. 
[20] Jorio A, Pimenta MA, Souza Filho AG, Samsonidze GG, Swan AK, Ünlü MS, Goldberg BB, Saito R, Dresselhaus G and Dresselhaus MS 2003 Phys. Rev. Lett. 90107403

[21] Sumanasekera GU, Allen JL, Fang SL, Loper AL, Rao AM and Eklund PC 1999 J. of Phys. Chem. B 1034292

[22] Rao AM, Bandow S, Richter E and Eklund PC 1998 Thin Solid Films 331141

[23] Hadjiev VG, Iliev MN, Arepalli S, Nikolaev P and Files BS 2001 Appl. Phys. Lett. 783193

[24] Seber and Wild, Nonlinear Regression 1989 John Wiley \& Sons 
FIG. 1: 3D optical microscope image of the line scan laser beam on the sample surface (a) and the corresponding Raman image (b) as recorded on the CCD detector. The line-scan crosses at least five SWCNTs/bundles as evidenced by the Raman spectra in the $1350-1850 \mathrm{~cm}^{-1}$.

FIG. 2: Typical Raman spectra of a SWCNTs bundle containing metallic nanotubes (strong $\omega_{G^{-}}^{-}$ band) and a semiconducting isolated nanotube (strong $\omega_{G}^{+}$-band). Note the difference in the scattering intensity.

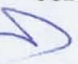

FIG. 4: Two dimensional Raman images of $13.5 \times 8,5 \mu m^{2}$ area on the sample displaying intensity distribution of (a) $\omega_{G}^{+}$-band, (b) $\omega_{G}^{-}$-band, and (c) D-band.

FIG. 5: Raman spectra covering the D- and G-band vibrations at the spots 1 - 5 in Fig. 5 (b). The spectra display characteristic lineshapes of an isolated SWCNT, 1-2, and those of bundles 3-5 with diffenet proportions of metallic to semiconducting nanotubes.

FIG. 6: Polarized $\omega_{G}^{+}$images (a) and (b) recorded for two orthogonal light polarizations denoted by the arrows in the figure. The SWCNTs' position reconconstruction is given in (c).

FIG. 3: $\omega_{G}^{+}$intensity profile along the line scan. 


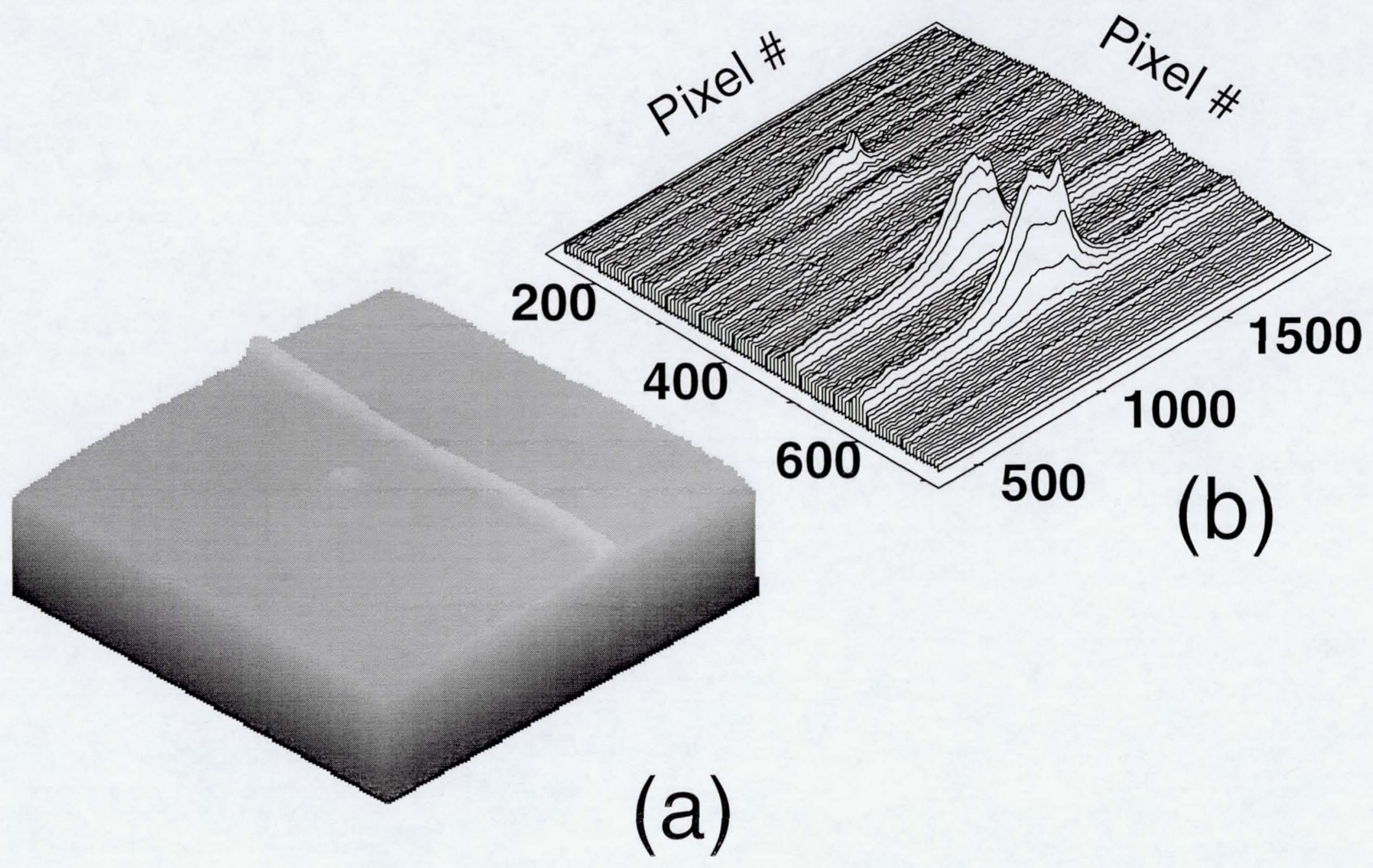




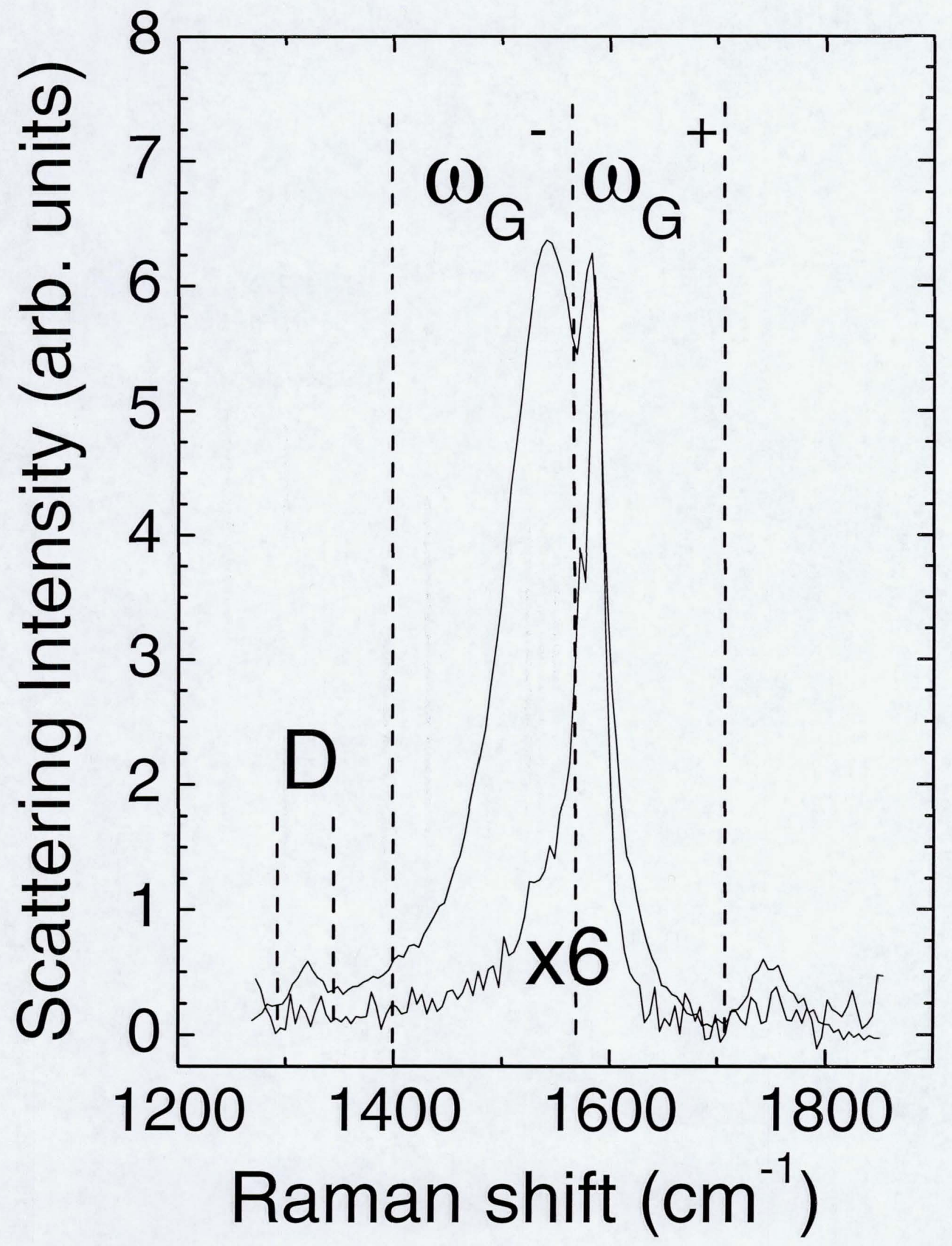




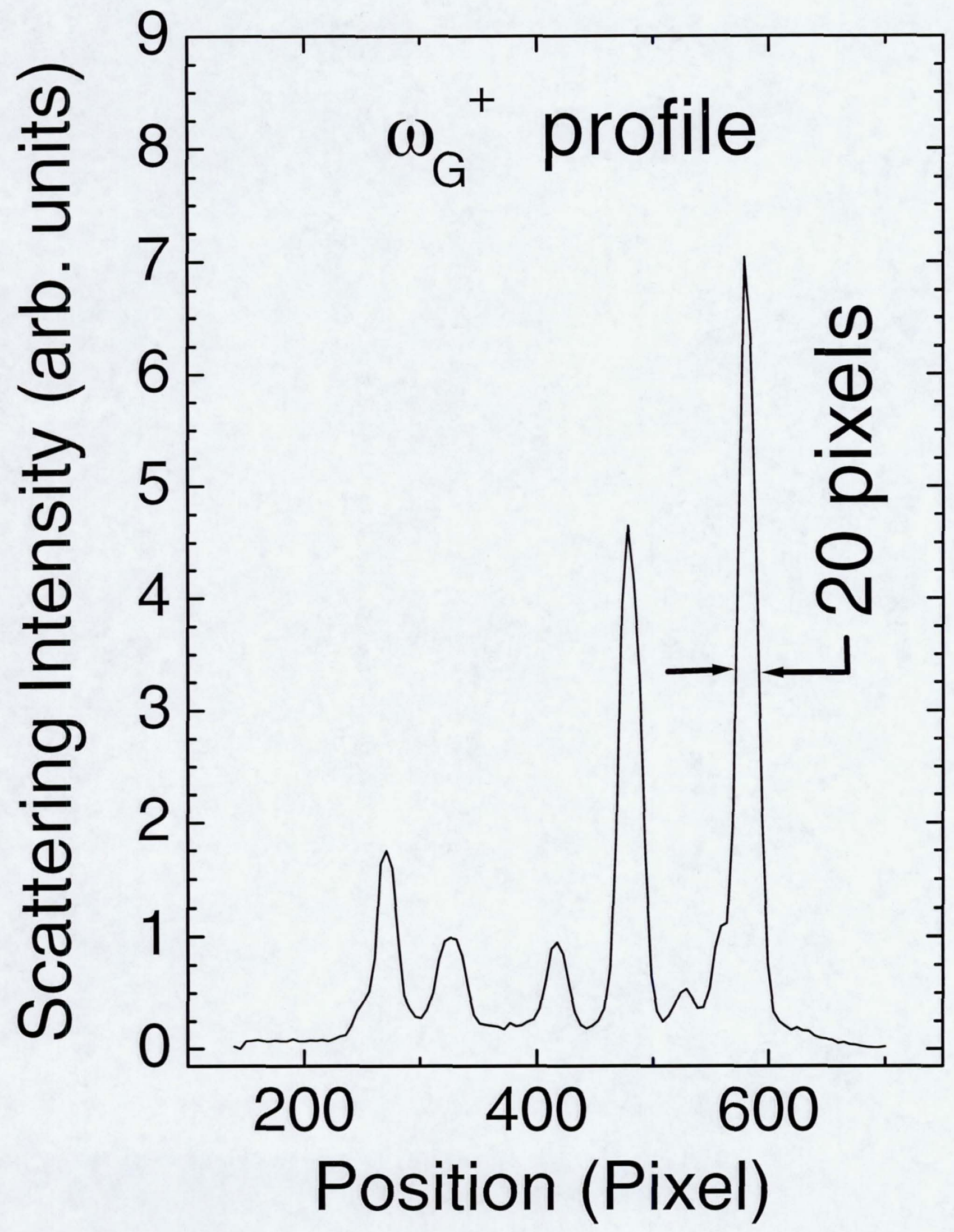



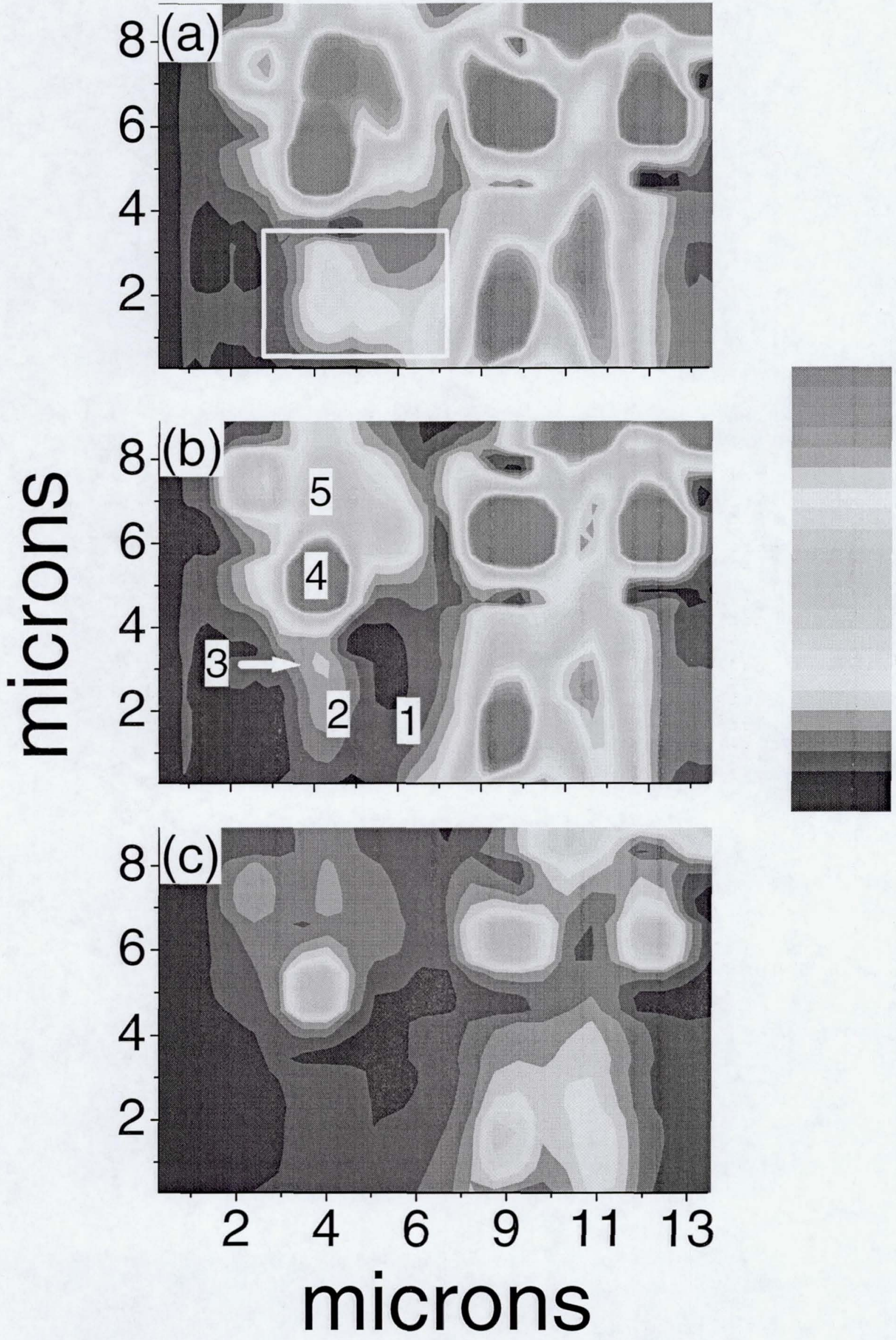


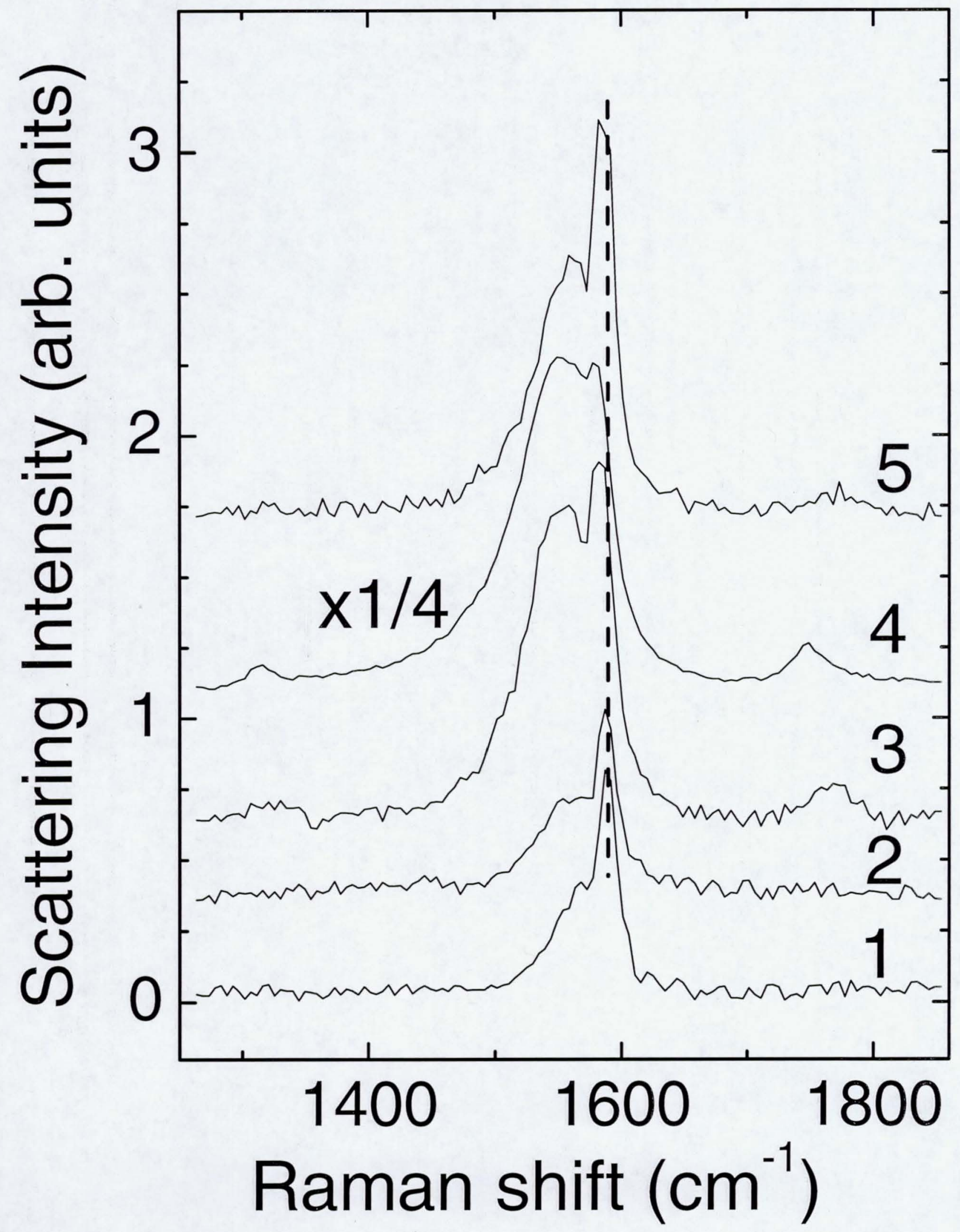




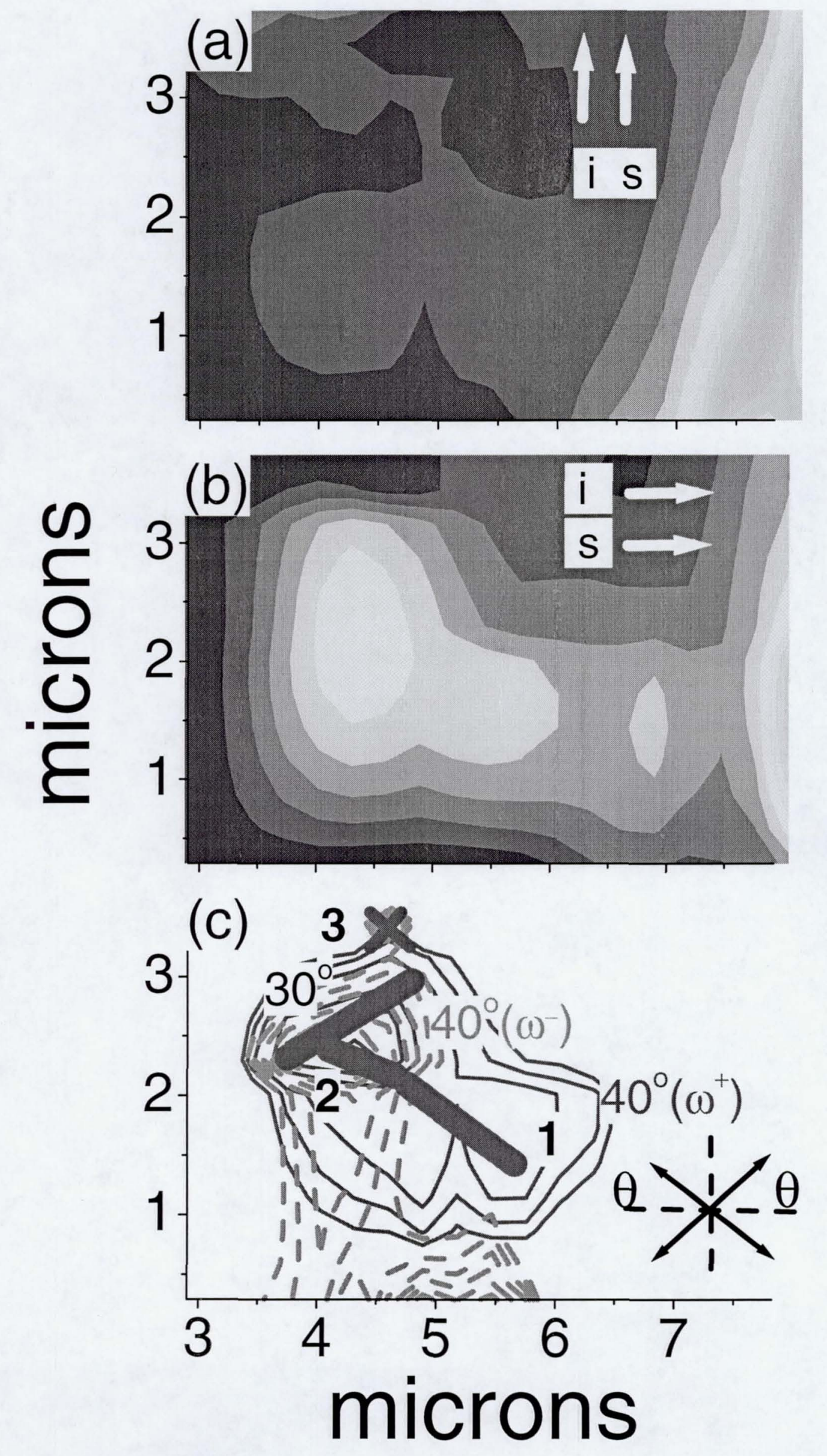

